\title{
ISOMETRY GROUPS OF SEPARABLE METRIC SPACES
}

\author{
MACIEJ MALICKI AND SŁAWOMIR SOLECKI
}

\begin{abstract}
We show that every locally compact Polish group is isomorphic to the isometry group of a proper separable metric space. This answers a question of Gao and Kechris. We also analyze the natural action of the isometry group of a separable ultrametric space on the space. This leads us to a structure theorem representing an arbitrary separable ultrametric space as a bundle with an ultrametric base and with ultrahomogeneous fibers which are invariant under the action of the isometry group.
\end{abstract}

\section{INTRODUCTION}

For a metric space $(X, d)$, let $\operatorname{Iso}(X)$ be the group of all isometries of $X$ equipped with the pointwise convergence topology.

The first part of the paper is concerned with representing groups as full isometry groups of metric spaces so that nice properties of the group are reflected by nice properties of the metric space. It is easy to see that if $X$ is a Polish metric space, then $\operatorname{Iso}(X)$ is Polish. Again, it is an easy observation that $\operatorname{Iso}(X)$ is compact provided that $X$ is compact. It was proved in [4] that $\operatorname{Iso}(X)$ is locally compact if $X$ is proper, that is, if all closed balls of $(X, d)$ are compact. A natural question arises whether the converses to these facts hold.

In [4], Gao and Kechris showed that every Polish group is indeed isomorphic to the isometry group of some Polish space. Then Melleray [7] found a simpler proof of their result and used it to prove that every compact group is isomorphic to the isometry group of a compact space. In Section 2 Theorem 2.1, we provide the last missing piece of the picture by showing that every locally compact Polish group is the isometry group of a proper Polish space. This solves a problem posed by Gao and Kechris in [4, p.76].

In Section 3, we comment on the tools used in the proof of the main result from the previous section.

2000 Mathematics Subject Classification. 22D12, 54E35, 54H15.

Key words and phrases. Isometry group, locally compact group, ultrametric space.

Research of Solecki was supported by NSF grant DMS-0700841.

Filename: heineborelfinal.tex. 
Then we turn to ultrametric spaces and their isometry groups. One can view Theorem 2.1 from Section 2 as characterizing isometry groups of proper Polish spaces. A similar problem, also mentioned in [4], is that of characterizing isometry groups of Polish ultrametric spaces. We touch on this problem in Section 4. First, we prove in Proposition 4.1 that the only non-trivial simple topological groups that are isometry groups of Polish ultrametric spaces are $\mathbb{Z}_{2}$ and $S_{\infty}$. This, combined with an observation that such groups are isomorphic to closed subgroups of $S_{\infty}$, gives a new argument that isometry groups of Polish ultrametric spaces form a proper subset of the family of closed subgroups of $S_{\infty}$ (see [4].) Further, we analyze the natural action of the isometry group of an ultrametric separable space on the space. This leads to a structure theorem, Theorem 4.2, for separable ultrametric spaces related to some results announced in [3]. This theorem represents each separable ultrametric space $X$ as a "bundle" with an ultrametric base and with fibers on which Iso $(X)$ acts ultrahomogeneously and which are equal to orbits of the action of Iso $(X)$ on $X$. We call the fibers homogeneity components. Theorem 4.2 also provides information about when isometries of homogeneity components of $X$ can be extended to isometries of $X$. Finally, we construct an example of a Polish ultrametric space with two homogeneity components and an isometry of one of them which cannot be extended to an isometry of the whole space.

Most of the notions used throughout this paper are standard. Otherwise, we formulate them explicitly. Recall, that a topological space $X$ is called Polish if it is separable and completely metrizable. Often, we will be interested in a particular metric $d$ on $X$ making it into a Polish space. Then we will call the pair $(X, d)$ a Polish metric space.

A mapping $\phi: X \rightarrow X$ is an isometry of $X$ if it is an isometric bijection. It is well-known that the full group of isometries of a Polish metric space $(X, d), \operatorname{Iso}(X, d)$, is a Polish group if equipped with the pointwise convergence topology. That is the only topology on $\operatorname{Iso}(X)$ that will be considered in this paper. If $d$ is clear from the context, we will write $X$ and $\operatorname{Iso}(X)$, instead of $(X, d)$ and $\operatorname{Iso}(X, d)$, respectively.

For a metric space $(X, d)$ and nonempty $A, B \subseteq X$, we define

$$
\operatorname{dist}(A, B)=\inf \{d(a, b): a \in A, b \in B\}
$$

Results of this paper were obtained as follows. Using methods outlined in Section 3, the first author proved a weaker version of Theorem 2.1 with $G$ additionally assumed to be uncountable and with a pseudo-connected space $Z$ in the conclusion. (Properness implies pseudo-connectedness; for the definition of pseudo-connectedness see [4, p.32].) Afterwards, the second 
author obtained the current version of Theorem 2.1. Section 4 constitutes joint work.

\section{LOCALLY COMPACT ISOMETRY GROUPS}

Recall that a metric space is called proper if all closed balls in it are compact.

Theorem 2.1. Let $G$ be a locally compact, second countable group. There exists a proper Polish metric space $Z$ with $G=\operatorname{Iso}(Z)$.

In relation to the thorem above, recall that, as proved in [4], if $Z$ is proper, then $\operatorname{Iso}(Z)$ is locally compact. For other general sufficient conditions on $Z$ guaranteeing local compactness of $\operatorname{Iso}(Z)$ the reader may consult [4] and [6].

Following [5] (see also [4, Section $2 \mathrm{C}]$ ), for a metric space $(X, d)$, we consider the space $E(X)$ of Katětov functions on $X$, that is, functions $f$ : $X \rightarrow \mathbb{R}$ satisfying

$$
|f(x)-f(y)| \leq d(x, y) \leq f(x)+f(y)
$$

for all $x, y \in X$. The set $E(X)$ is made into a metric space with the metric defined by

$$
\sup _{x \in X}|f(x)-g(x)| .
$$

Since this metric extends $d$ if we identify elements $x \in X$ with their distance functions $d(x, \cdot) \in E(X)$, we will denote it again by $d$. In general, we have that

$$
d(f, x)=f(x)
$$

for $f \in E(X)$ and $x \in X$. Given an isometry $\phi$ of $X$, by $\phi^{*}$ we denote the induced isometry of $E(X)$ given by

$$
\phi^{*}(f)=f\left(\phi^{-1}(x)\right) .
$$

The extension $\phi^{*}$ restricted to $X$, viewed as a subspace of $E(X)$, is equal to $\phi$. Furthermore note that if $f \in E(X)$ and

$$
\phi: X \cup\{f\} \rightarrow E(X)
$$

is an isometric embedding and $\phi(X)=X$, then

$$
\phi=(\phi \uparrow X)^{*} \uparrow(X \cup\{f\}) .
$$

Indeed, by (1), for an arbitrary $x \in X$ we have

$$
\phi(f)(x)=d(\phi(f), x)=d\left(f, \phi^{-1}(x)\right)=f\left(\phi^{-1}(x)\right) .
$$


We will be interested in certain subspaces of $E(X)$. Define for $n \in \mathbb{N}$, $n \geq 1, E_{n}^{\prime}(X)$ to be the space of all those $f: X \rightarrow \mathbb{R}$ for which for some $x_{1}, \ldots, x_{n} \in X$ we have

(3) $f\left(x_{i}\right)+f\left(x_{j}\right) \geq d\left(x_{i}, x_{j}\right),\left|f\left(x_{i}\right)-f\left(x_{j}\right)\right| \leq \frac{1}{3} d\left(x_{i}, x_{j}\right)$ for $1 \leq i, j \leq n$, and

$$
f(x)=\min _{1 \leq i \leq n} f\left(x_{i}\right)+d\left(x, x_{i}\right) .
$$

Note that functions in $E_{n}^{\prime}(X)$ are Katětov, so $E_{n}^{\prime}(X)$ is a subspace of $E(X)$.

Two general lemmas about $E_{n}^{\prime}(X)$, which we now prove, will be useful in the proof of Theorem 2.1.

Lemma 2.2. If $X$ is proper, then so is $E_{n}^{\prime}(X)$.

Proof. Fix $\bar{x} \in X$. It will suffice to show that

$$
A_{r}=\left\{f \in E_{n}^{\prime}(X): f(\bar{x}) \leq r\right\} \text { is compact }
$$

for any $r>0$. Let $f \in A_{r}$ and let $x_{1}, \ldots, x_{n} \in X$ be as in the definition of $E_{n}^{\prime}(X)$ chosen for $f$, that is, fulfilling (3) and (4). Note first that for all $1 \leq i, j \leq n$

$$
\frac{1}{3} d\left(x_{i}, x_{j}\right) \leq \min \left\{f\left(x_{i}\right), f\left(x_{j}\right)\right\} .
$$

To see this, observe that since $f \in E_{n}^{\prime}(X)$, we have

$$
d\left(x_{i}, x_{j}\right) \leq f\left(x_{i}\right)+f\left(x_{j}\right) \leq f\left(x_{i}\right)+f\left(x_{i}\right)+\frac{1}{3} d\left(x_{i}, x_{j}\right),
$$

hence $(2 / 3) d\left(x_{i}, x_{j}\right) \leq 2 f\left(x_{i}\right)$, and (5) follows.

Now fix $\bar{i} \in\{1, \ldots, n\}$ so that

$$
f(\bar{x})=f\left(x_{\bar{i}}\right)+d\left(\bar{x}, x_{\bar{i}}\right) .
$$

Then,

$$
d\left(\bar{x}, x_{\bar{i}}\right) \leq f\left(x_{\bar{i}}\right)+d\left(\bar{x}, x_{\bar{i}}\right)=f(\bar{x}) \leq r,
$$

and by (5) for any $j \in\{i, \ldots, n\}$

$$
\frac{1}{3} d\left(x_{\bar{i}}, x_{j}\right) \leq f\left(x_{\bar{i}}\right) \leq f\left(x_{\bar{i}}\right)+d\left(\bar{x}, x_{\bar{i}}\right)=f(\bar{x}) \leq r .
$$

Thus, by (6) and (7) we get

$$
\max _{1 \leq j \leq n} d\left(\bar{x}, x_{j}\right) \leq 4 r .
$$

Since $X$ is proper, the ball $\{x \in X: d(\bar{x}, x) \leq 4 r\}$ is compact, and compactness of $A_{r}$ follows. 
Lemma 2.3. Let $(X, d)$ be a metric space and let $f: X \rightarrow \mathbb{R}$ and $x_{1}, x_{2} \in X$ be such that

$$
f(x)=\min \left\{f\left(x_{1}\right)+d\left(x, x_{1}\right), f\left(x_{2}\right)+d\left(x, x_{2}\right)\right\}
$$

and

$$
f\left(x_{1}\right)+f\left(x_{2}\right)>d\left(x_{1}, x_{2}\right) \text { and } 0<\left|f\left(x_{1}\right)-f\left(x_{2}\right)\right|<\frac{1}{3} d\left(x_{1}, x_{2}\right) .
$$

Let $\delta>0$ be small enough. If $\left\{x \in X: d\left(x, x_{1}\right) \leq \delta\right\}$ and $\left\{x \in X: d\left(x, x_{2}\right) \leq\right.$ $\delta\}$ contain sets of size $k \in \mathbb{N}, k \geq 1$, of points at distance $\geq \delta$ from each other, then

$$
\left\{h \in E_{1+k}^{\prime}(X): \min h=\min f \text { and } d(h, f) \leq \delta\right\}
$$

contains such a set of size $2^{k}$.

Proof. Since the assumptions on $x_{1}$ and $x_{2}$ are symmetric and $f\left(x_{1}\right) \neq f\left(x_{2}\right)$, we can suppose that $f\left(x_{1}\right)>f\left(x_{2}\right)$. Let $y_{1}, \ldots, y_{k} \in\left\{x \in X: d\left(x, x_{1}\right) \leq \delta\right\}$ be such that $d\left(y_{i}, y_{j}\right) \geq \delta$ if $i \neq j$. For $I \subseteq\{1, \ldots, k\}$ with $I \neq \emptyset$, let

$$
h_{I}(x)=\min \left\{\min _{i \in I}\left\{f\left(x_{1}\right)+d\left(x, y_{i}\right)\right\}, f\left(x_{2}\right)+d\left(x, x_{2}\right)\right\} .
$$

For $I=\emptyset$, let

$$
h_{\emptyset}(x)=\min \left\{f\left(x_{1}\right)-\delta+d\left(x, x_{1}\right), f\left(x_{2}\right)+d\left(x, x_{2}\right)\right\} .
$$

We claim that if $I \neq J$ are subsets of $\{1, \ldots, k\}$, then

$$
d\left(h_{I}, h_{J}\right) \geq \delta
$$

If $I, J$ are both non-empty, we can fix $i \in I \triangle J$, say $i \in I \backslash J$. Note that for any $j \neq i$

$$
f\left(x_{1}\right)+d\left(y_{i}, y_{j}\right) \geq f\left(x_{1}\right)+\delta .
$$

and, since $\delta>0$ is small, that

$$
f\left(x_{2}\right)+d\left(y_{i}, x_{2}\right) \geq f\left(x_{2}\right)+d\left(x_{1}, x_{2}\right)-\delta \geq f\left(x_{1}\right)+\delta
$$

Thus,

$$
h_{J}\left(y_{i}\right) \geq f\left(x_{1}\right)+\delta \text { while } h_{I}\left(y_{i}\right)=f\left(x_{1}\right)
$$

and, therefore, (8) holds. If $I=\emptyset$ and $J \neq \emptyset$, then $h_{\emptyset}\left(x_{1}\right)=f\left(x_{1}\right)-\delta$ while $h_{J}\left(x_{1}\right) \geq f\left(x_{1}\right)$; thus, again (8) holds.

Further, using the inequality

$$
\left|\min _{1 \leq s \leq l} a_{s}-\min _{1 \leq s \leq l} b_{s}\right| \leq \max _{1 \leq s \leq l}\left|a_{s}-b_{s}\right|,
$$


we get for non-empty $I$

$$
\begin{aligned}
\left|h_{I}(x)-f(x)\right| \leq & \max \left\{\max _{i \in I}\left|f\left(x_{1}\right)+d\left(x, y_{i}\right)-\left(f\left(x_{1}\right)+d\left(x, x_{1}\right)\right)\right|,\right. \\
& \left.\left|f\left(x_{2}\right)+d\left(x, x_{2}\right)-\left(f\left(x_{2}\right)+d\left(x, x_{2}\right)\right)\right|\right\} \\
& \leq \max _{i \in I}\left|d\left(x, y_{i}\right)-d\left(x, x_{1}\right)\right| \leq \max _{i \in I} d\left(y_{i}, x_{1}\right) \leq \delta,
\end{aligned}
$$

and similarly

$$
\begin{array}{r}
\left|h_{\emptyset}(x)-f(x)\right| \leq \max \left\{\left|f\left(x_{1}\right)-\delta+d\left(x, x_{1}\right)-\left(f\left(x_{1}\right)+d\left(x, x_{1}\right)\right)\right|,\right. \\
\left.\left|f\left(x_{2}\right)+d\left(x, x_{2}\right)-\left(f\left(x_{2}\right)+d\left(x, x_{2}\right)\right)\right|\right\}=\delta .
\end{array}
$$

Thus, for each $I \subseteq\{1, \ldots, k\}$,

$$
d\left(h_{I}, f\right) \leq \delta .
$$

Since there are $2^{k}$ subsets of $\{1, \ldots, k\}$, we will be done by (8) and (10), if we only show that each $h_{I}$ is in $E_{1+k}^{\prime}(X)$ and that $\min h_{I}=\min f$. The latter assertion is clear since, by smallness of $\delta, \min h_{I}=f\left(x_{2}\right)=\min f$. The former one, is clear for $I=\emptyset$ by smallness of $\delta$. To check it for $I \neq \emptyset$, put $h=h_{I}$. One only needs to verify the following inequalities for $i, i^{\prime} \in I$

(i) $h\left(y_{i}\right)+h\left(y_{i^{\prime}}\right) \geq d\left(y_{i}, y_{i^{\prime}}\right)$;

(ii) $h\left(y_{i}\right)+h\left(x_{2}\right) \geq d\left(y_{i}, x_{2}\right)$;

(iii) $\left|h\left(y_{i}\right)-h\left(y_{i^{\prime}}\right)\right| \leq(1 / 3) d\left(y_{i}, y_{i^{\prime}}\right)$;

(iv) $\left|h\left(y_{i}\right)-h\left(x_{2}\right)\right| \leq(1 / 3) d\left(y_{i}, x_{2}\right)$.

As computed by (9), $h\left(y_{i}\right)=h\left(y_{i^{\prime}}\right)=f\left(x_{1}\right)$. Since $\delta>0$ is small, we also get $h\left(x_{2}\right)=f\left(x_{2}\right)$. Thus, (i)-(iv) follow from our assumptions on $f$ and from smallness of $\delta$.

Proof of Theorem 2.1. It is a folklore observation that any locally compact Polish group $G$ admits a compatible left-invariant proper metric, which is automatically complete. Its existence is established by following the proof of the Birkhoff-Kakutani theorem and the fact that there exists in such a $G$ a sequence $\left(V_{k}\right)_{k \in \mathbb{Z}}$ of compact symmetric neighborhoods of the identity 1 such that $V_{k}^{3} \subseteq V_{k+1}, \bigcap_{k} V_{k}=\{1\}$, and $\bigcup_{k} V_{k}=G$.

Fix such a metric $d$ on $G$. We denote by the same letter the extension of $d$ to the space of Katětov functions $E(G)$. Note that each element $g_{0}$ of $G$ induces an isometry of $G$ by

$$
G \in g \rightarrow g_{0} g \in G .
$$

This provides a homeomorphic and homomorphic embedding of $G$ into Iso $(G)$. From this point on we will consider $G$ as a subgroup of $\operatorname{Iso}(G)$.

We construct now functions $f_{i}, h_{i} \in E(G)$ and $\epsilon_{i}>0$, for $i \geq 1$, so that

(a) the family $\left\{\phi \in \operatorname{Iso}(G): d\left(\phi^{*}\left(f_{i}\right), h_{i}\right)<\epsilon_{i} / 2\right\}$ with $i \in \mathbb{N}, i \geq 1$, covers $\operatorname{Iso}(G) \backslash G$; 
(b) $\left\{\phi \in \operatorname{Iso}(G): d\left(\phi^{*}\left(f_{i}\right), h_{i}\right)<\epsilon_{i}\right\}$ is disjoint from $G$ for each $i$;

(c) $0<\min f_{i}<\min f_{i+1} \rightarrow \infty$ as $i \rightarrow \infty$;

(d) $f_{i}$ is as in the assumptions of Lemma 2.3.

To define the objects with properties (a)-(d), we start with the following claim.

Claim 1. Let $\psi \in \operatorname{Iso}(G) \backslash G$. There exist $x_{1}, x_{2} \in G$ and $n>0$ such that

$$
U_{\psi, n}=\left\{\phi \in \operatorname{Iso}(G): d\left(\phi\left(x_{1}\right), \psi\left(x_{1}\right)\right), d\left(\phi\left(x_{2}\right), \psi\left(x_{2}\right)\right)<1 / n\right\}
$$

is disjoint from $G$.

Proof of Claim 1. Set $x_{1}$ to be any element of $G$ and let $g \in G$ be such that $g x_{1}=\psi\left(x_{1}\right)$. Since $\psi \notin G$ there is $x_{2} \in G$ with $g x_{2} \neq \psi\left(x_{2}\right)$.

If for every $n>0$ there exists $g_{n} \in G$ with

$$
d\left(g_{n} x_{1}, \psi\left(x_{1}\right)\right), d\left(g_{n} x_{2}, \psi\left(x_{2}\right)\right)<1 / n
$$

then $g_{n} x_{1} \rightarrow g x_{1}$, so $g_{n} \rightarrow g$. But then $g_{n} x_{2} \rightarrow g x_{2} \neq \psi\left(x_{2}\right)$ which contradicts (11). This finishes the proof of the claim.

Given $\psi$ as in the assumptions of Claim 1, fix $x_{1}, x_{2}$ and $n$ as given by the conclusion of that claim. For $M \geq d\left(x_{1}, x_{2}\right) / 2$ define

$$
\begin{aligned}
& f_{M}(x)=\min \left\{M+d\left(x, x_{1}\right), M+\frac{1}{4} d\left(x_{1}, x_{2}\right)+d\left(x, x_{2}\right)\right\} \\
& h_{M}(x)=\min \left\{M+d\left(x, \psi\left(x_{1}\right)\right), M+\frac{1}{4} d\left(x_{1}, x_{2}\right)+d\left(x, \psi\left(x_{2}\right)\right)\right\} .
\end{aligned}
$$

It is easy to check that for $\epsilon>0$ small enough we have

$$
\left\{\phi \in \operatorname{Iso}(G): d\left(\phi^{*}\left(f_{M}\right), h_{M}\right)<\epsilon\right\} \subseteq U_{\psi, n},
$$

that $\min f_{M} \geq M$ and that $f_{M}$ fulfills the assumptions of Lemma 2.3. Using the observations above, it is routine to construct $f_{i}, h_{i}, \epsilon_{i}>0$ with (a)-(d) as desired.

Now, for each $i \geq 1$, pick $\delta_{i}>0$ so that

(i) some (each) closed $d$-ball in $G$ of radius $\delta_{i}$ is compact;

(ii) $\delta_{i}<\epsilon_{i} / 2$;

(iii) $\delta_{i}<\min \left\{\min f_{i}: i \in \mathbb{N}\right\} / 2$;

(iv) $\delta_{i}$ is small enough for $f_{i}$ so that the conclusion of Lemma 2.3 holds.

Point (i) allows us to pick $k_{i} \in \mathbb{N}, k_{i} \geq 1$, so that $k_{i}$ is the maximal number of elements in a closed $d$-ball in $G$ of radius $\delta_{i}$ that are at distance $\geq \delta_{i}$ from each other. By Lemma 2.3, there are $h_{i}^{s}, 1 \leq s \leq 2^{k_{i}}$, such that

( $\alpha) h_{i}^{s} \in E_{1+k_{i}}^{\prime}(G)$ and $\min h_{i}^{s}=\min f_{i}$;

(ß) $d\left(h_{i}^{s}, f_{i}\right) \leq \delta_{i}$

$(\gamma) d\left(h_{i}^{s}, h_{i}^{t}\right) \geq \delta_{i}$ if $s \neq t$. 
Define $Z_{0}=G$ and for $i \geq 1$

$$
Z_{i}=\left\{g^{*}\left(f_{i}\right): g \in G\right\} \cup\left\{g^{*}\left(h_{i}^{s}\right): 1 \leq s \leq 2^{k_{i}}, g \in G\right\} .
$$

Let $Z=\bigcup_{i \in \mathbb{N}} Z_{i}$.

Note that by $(\mathrm{d}), g^{*}\left(f_{i}\right) \in E_{2}^{\prime}(G) \subseteq E_{1+k_{i}^{\prime}}(G)$ for each $g \in G$. Thus, $Z_{i} \subseteq E_{1+k_{i}^{\prime}}(G)$ for each $i \geq 1$. Therefore, by Lemma 2.2, (c), and $(\alpha), Z$ with the metric $d$ is proper. We claim that $G=\operatorname{Iso}(Z)$. Define a function from $G$ to $\operatorname{Iso}(Z)$ by

$$
G \ni g \rightarrow g^{*} \uparrow Z \in \operatorname{Iso}(Z) .
$$

It is easy to check that this is a continuous injective group homomorphism. We show that it is onto, which will prove the theorem.

Claim 2. If $\phi \in \operatorname{Iso}(Z)$, then $\phi\left(Z_{i}\right) \cap G=\emptyset$ for each $i \geq 1$.

Proof of Claim 2. Assume that this is not the case, that is, for some $i \geq 1$ and some $g \in G$ we have

$$
\phi\left(g^{*}\left(f_{i}\right)\right) \in G \text { or } \phi\left(g^{*}\left(h_{i}^{s}\right)\right) \in G \text { for some } 1 \leq s \leq 2^{k_{i}} .
$$

Since $d\left(g^{*}\left(f_{i}\right), g^{*}\left(h_{i}^{s}\right)\right) \leq \delta_{i}$ and $\operatorname{dist}\left(Z_{j}, G\right)>\delta_{i}$ for $j \geq 1$, we have that

$$
\phi\left(g^{*}\left(f_{i}\right)\right) \in G \text { and } \phi\left(g^{*}\left(h_{i}^{s}\right)\right) \in G \text { for all } 1 \leq s \leq 2^{k_{i}} .
$$

Thus, the set

$$
\left\{\phi\left(g^{*}\left(h_{i}^{s}\right)\right): 1 \leq s \leq 2^{k_{i}}\right\}
$$

is contained in the closed $d$-ball in $G$ centered at $\phi\left(g^{*}\left(f_{i}\right)\right) \in G$ and of radius $\delta_{i}$. The points in this set are at distance $\geq \delta_{i}$ from each other and there are $2^{k_{i}}$ of them. Since $2^{k_{i}}>k_{i}$, this contradicts the choice of $k_{i}$ and proves the claim.

Let $\phi \in \operatorname{Iso}(Z)$. It follows from Claim 2 that $\phi(G) \supseteq G$. By applying Claim 2 to $\phi^{-1}$, we see that $\phi^{-1}(G) \supseteq G$, hence $\phi(G) \subseteq G$. It follows that $\phi(G)=G$. Thus, by the discussion at the beginning of this section and, in particular, by formula (2),

$$
\phi=\psi^{*} \uparrow Z \text { for } \psi=\phi \uparrow G .
$$

Further, since for $h \in Z_{i}$ and $h^{\prime} \in Z_{j}$ with $i, j \geq 1, i \neq j$ we have by $(\alpha)$ and $(\mathrm{c})$

$$
\operatorname{dist}(h, G)=\operatorname{dist}\left(Z_{i}, G\right) \neq \operatorname{dist}\left(Z_{j}, G\right)=\operatorname{dist}\left(h^{\prime}, G\right),
$$

we get $\psi^{*}\left(Z_{i}\right)=Z_{i}$ for each $i$. Thus, $\psi^{*}\left(f_{i}\right) \in Z_{i}$, so $d\left(\psi^{*}\left(f_{i}\right), g^{*}\left(f_{i}\right)\right) \leq \delta_{i}$ for some $g \in G$. Since $\delta_{i}<\epsilon_{i} / 2$ and, as follows from (b), $d\left(g^{*}\left(f_{i}\right), h_{i}\right) \geq \epsilon_{i}$, we get

$$
d\left(\psi^{*}\left(f_{i}\right), h_{i}\right)>\epsilon_{i} / 2 .
$$

Since this happens for each $i$, by (a), we have $\psi=g_{0} \in G$, hence $\phi=g_{0}^{*} \uparrow Z$ as required. 


\section{COMments ON LOCALly COMPACT ISOMETRY GROUPS}

A crucial element of the proof of Theorem 2.1 are properly chosen subspaces of $E(X)$. Originally, we took a somewhat different approach that lead to a weaker version of Theorem 2.1 (for uncountable $G$ and with proper metric spaces replaced by a weaker notion of pseudo-connected metric spaces, see [4] for definition). In this approach we used different subspaces and new metrics on them. These metrics may be of some independent interest, so we will briefly sketch their main properties.

If, for a given $f \in E(X)$, there exists $S \subseteq X$ such that

$$
f(x)=\min \{f(s)+d(x, s): s \in S\}
$$

for any $x \in X$, we say that $S$ is a support of $f$. If there exists a compact (finite) $S$ as above, we say that $f$ is a Katětov function with compact (finite) support. (Katětov's functions with finite support play an important role in [5].)

Lemma 3.1. Suppose that $f$ is a Katětov function with compact support. Then there exists a smallest support for $f$, denoted by $S(f)$, that is, a set supporting $f$ which is contained in every other support of $f$.

Proof. First we consider the case that $f$ has finite support. Pick a finite set $S$ supporting $f$. If

$$
f\left(s_{1}\right)=f\left(s_{2}\right)+d\left(s_{1}, s_{2}\right)
$$

for some $s_{1}, s_{2} \in S$, then $S \backslash\left\{s_{1}\right\}$ is also supporting $f$. By removing points from $S$, we find a supporting set $S_{0}$ such that

$$
\forall s_{1}, s_{2} \in S_{0} f\left(s_{1}\right) \neq f\left(s_{2}\right)+d\left(s_{1}, s_{2}\right) .
$$

We claim that, for any supporting $T, S_{0} \subseteq T$. If not, there is $s_{1} \in S_{0} \backslash T$ and $t \in T \backslash S_{0}$ with

$$
f\left(s_{1}\right)=f(t)+d\left(s_{1}, t\right) .
$$

But

$$
f(t)=f\left(s_{2}\right)+d\left(t, s_{1}\right)
$$

for some $s_{2} \in S_{0}$, so

$$
f\left(s_{1}\right)=f\left(s_{2}\right)+d\left(t, s_{1}\right)+d\left(t, s_{2}\right) \geq f\left(s_{1}\right)+d\left(s_{1}, s_{2}\right) .
$$

The fact that $f$ is Katětov implies that

$$
f\left(s_{1}\right)=f\left(s_{2}\right)+d\left(s_{1}, s_{2}\right),
$$

which contradicts the definition of $S_{0}$.

For the general case, consider a sequence $\left\{f_{n}\right\}$ of functions with finite support converging to $f$. 
Suppose that $d^{\prime}$ is another compatible metric on $X$. In light of the preceding lemma, we can define the following metric $\rho$ on the set of Katětov functions on $X$ with compact support:

$$
\rho(f, g)=\sup _{x}\{|f(x)-g(x)|\}+d^{\prime}(S(f), S(g))
$$

It is easy to see that this space, denoted by $E_{C}\left(X, d, d^{\prime}\right)$, is Polish.

In the proof of the next proposition, the symbol $\mathcal{K}(X)$ stands for the space of all compact subsets of $X$, with the Vietoris topology. For a metric space $(X, d), \bar{B}_{d}(x, r)$ is a closed ball in $X$, centered at $x$, and with radius $r$.

Proposition 3.2. Assume that $X$ is a locally compact space, and let $d$, $d^{\prime}$ be compatible metrics on $X$. Then $E_{C}\left(X, d, d^{\prime}\right)$ is also locally compact. Moreover, if $d^{\prime}$ is proper, so is $E_{C}\left(X, d, d^{\prime}\right)$.

Proof. Denote by $d^{\prime \prime}$ the Hausdorff metric on $\mathcal{K}(X)$ induced by $d^{\prime}$. Let $f \in$ $E_{C}\left(X, d, d^{\prime}\right)$, and let $r>0$ be such that $\bar{B}_{d^{\prime \prime}}(S(f), r)$ is compact. Suppose $\left\{f_{n}\right\}$ is a sequence contained in $\bar{B}_{\rho}(f, r)$. Since $S\left(f_{n}\right) \subseteq \bar{B}_{d^{\prime \prime}}(S(f), r)$ and the space of all compact subsets of a compact space is always compact, we can assume without loss of generality that $S\left(f_{n}\right)$ converge to some compact $S \subseteq \bar{B}_{d^{\prime \prime}}(S(f), r)$. Thus, it is enough to prove that $\left\{f_{n}\right\}$ restricted to $S$ contains a convergent subsequence. But this follows from Ascoli's theorem: $\left\{f_{n}\right\}$ is equicontinuous since the $f_{n}$ are Katětov, and are pointwise bounded by $f(s)+r$, for any $s \in S$. Hence, $\bar{B}_{\rho}(f, r)$ is compact.

The other statement follows by the same argument, because if $d^{\prime}$ is proper, then $\bar{B}_{d^{\prime}}(S(f), r)$ is compact for any $r>0$.

Clearly, $X$ can be topologically identified with the subset of $E_{C}\left(X, d, d^{\prime}\right)$ consisting of all distance functions $d(x, \cdot)$. The next lemma shows that if $X$ has no isolated points, and we choose $d^{\prime}$ in an appropriate way, isometries of $X$ can be uniquely extended to isometries of $E_{C}\left(X, d, d^{\prime}\right)$. In particular, Iso $(X)$ can be thought of as a closed subgroup of $\operatorname{Iso}\left(E_{C}\left(X, d, d^{\prime}\right)\right)$. We do not know whether this is true for $d=d^{\prime}$.

Proposition 3.3. Assume that $X$ has no isolated points. Then, for a given $\phi \in \operatorname{Iso}(X)$, the function $\phi^{*}$ defined by

$$
\phi^{*}(f)(x)=f\left(\phi^{-1}(x)\right)
$$

is the unique extension of $\phi$ to an isometry of $E_{C}\left(X, d, d^{1 / 2}\right)$, and the function $\phi \mapsto \phi^{*}$ is a continuous embedding of $\operatorname{Iso}(X)$ into $\operatorname{Iso}\left(E_{C}\left(X, d, d^{1 / 2}\right)\right)$.

Proof. Checking that $\phi^{*}$ is an isometry boils down to checking the equality $S\left(f\left(\phi^{-1}\right)\right)=\phi(S(f))$, which is straightforward.

To show uniqueness, it is enough to prove that if $\psi \in \operatorname{Iso}\left(E_{C}\left(X, d, d^{1 / 2}\right)\right)$ is such that $\psi(x)=x$ for every $x \in X$, then it is actually the identity. 
Fix such $\psi$ and let $\psi(f)=g$ for some $f$ with finite support. We have that

$$
\begin{aligned}
& \rho(f, x)=f(x)+d(S(f), x)^{\frac{1}{2}} \\
& \rho(g, x)=g(x)+d(S(g), x)^{\frac{1}{2}},
\end{aligned}
$$

that is,

$$
f(x)+d(S(f), x)^{\frac{1}{2}}=g(x)+d(S(g), x)^{\frac{1}{2}},
$$

or

$$
f(x)-g(x)=d(S(g), x)^{\frac{1}{2}}-d(S(f), x)^{\frac{1}{2}},
$$

for all $x \in X$.

First of all, notice that if $S(f) \neq S(g)$, then neither of them is a subset of the other. Otherwise, for $t$, say, an element of $S(g) \backslash S(f)$ and appropriate $s \in S(f)$, we have $f(s)=g(s)$, and

$$
f(t)=g(s)+d(t, s)+d(S(f), t)^{\frac{1}{2}}=g(t),
$$

implying that

$$
g(s)+d(t, s)<g(t),
$$

which contradicts that $g$ is Katětov.

If $S(f)=S(g)$, we are done. Assume there is $s \in S(f) \backslash S(g)$, and fix a ball $B \ni s$ such that

$$
\begin{aligned}
& d(x, S(f))=d(x, s), \text { for all } x \in B, \text { and } \\
& \inf _{x \in B} d(x, S(g)) \geq \delta>0 .
\end{aligned}
$$

Then, for $x \in B$,

$$
d(x, t)^{\frac{1}{2}}=d(x, S(f))^{\frac{1}{2}}=f(x)-g(x)+d(x, S(g))^{\frac{1}{2}} .
$$

Now, the right-hand side is Lipschitz on $B$, since $B \ni x \mapsto d(x, S(g))^{\frac{1}{2}}$ is Lipschitz as the composition of two Lipschitz functions $\left(r \mapsto r^{\frac{1}{2}}\right.$ is Lipschitz for $r>\delta$, and $f, g$ are Lipschitz being Katětov). On the other hand, the left-hand side is not Lipschitz, as there is a sequence $x_{n} \in B \backslash\{s\}$ with $x_{n} \rightarrow t$, contradiction.

The above argument shows that $\psi$ is the identity on the dense subset of $E_{C}\left(X, d, d^{\prime}\right)$, so it must be identity on the whole $E_{C}\left(X, d, d^{\prime}\right)$.

Continuity of $\phi \mapsto \phi^{*}$ can be easily established using the assumption that elements of $E_{C}\left(X, d, d^{\prime}\right)$ have compact support. Thus, it is an embedding. 


\section{Ultrametric SPACES}

Recall that a metric space $X$ is called ultrametric if it satisfies a strong version of the triangle inequality:

$$
d(x, z) \leq \max \{d(x, y), d(y, z)\}
$$

for every $x, y, z \in X$.

It is indicated in $[4$, p.30] that isometry groups of ultrametric Polish spaces are isomorphic to closed subgroups of the group $S_{\infty}$ of all permutations of $\mathbb{N}$. In $[4$, Proposition 4.7] the authors show that not all closed subgroups of $S_{\infty}$ are of this form by observing that every non-trivial isometry group of a Polish ultrametric space contains an element of order 2. In the proposition below, we give another restriction on isometry groups of Polish ultrametric spaces.

A topological group is called simple if it has no non-trivial closed normal subgroups. For $m \in \mathbb{N} \cup\{\infty\}$, by $S_{m}$ we denote the permutation group of a set of size $m$ if $m$ is finite, and of countable infinite size if $m=\infty$.

Proposition 4.1. $S_{\infty}$ and $\mathbb{Z}_{2}$ are the only simple non-trivial Polish groups isomorphic to the isometry group of an ultrametric space.

Proof. Let $G=\operatorname{Iso}(X, d)$ for an ultrametric space $(X, d)$ and put

$$
G_{r}=\{\phi \in G: \forall x \in X d(x, \phi(x)) \leq r\},
$$

for $r>0$. It is clear that $G_{r}$ is a closed normal subgroup of $G$.

Suppose that $G$ is a non-trivial, topologically simple Polish group, and $\phi_{0}\left(x_{0}\right)=y_{0}$ for some $\phi_{0} \in G$, and $x_{0}, y_{0} \in X$ with $r_{0}=d\left(x_{0}, y_{0}\right)>0$. We claim that for no $r_{1}<r_{0}$ there exists $\phi_{1} \in G$ and $x_{1}, y_{1} \in X$ with $\phi_{1}\left(x_{1}\right)=y_{1}$ and $d\left(x_{1}, y_{1}\right)=r_{1}$. Otherwise, we define $\psi \in G_{r_{1}}$ by putting $\psi(x)=\phi_{1}(x)$ if $x \in B$, and $\psi(x)=x$ for $x \notin B$, where $B$ is a closed ball centered at $x_{1}$, and of radius $r_{0}$. The isometry $\psi$ witnesses that $G_{r_{1}}$ is not trivial and $\phi_{0} \notin G_{r_{1}}$, which contradicts our assumption that $G$ is simple.

Thus, in each orbit of the action of $\operatorname{Iso}(X)$ on $X$, any two points are at distance $r_{0}$. Let $\left\{X_{n}: n \in \mathbb{N}\right\}$ be a maximal family of such orbits, satisfying $\operatorname{dist}\left(X_{n}, X_{m}\right)>r_{0}$ if $n \neq m$. It is not hard to see that, for $M_{n}$ being the size of $X_{n}$, we have that $G$ is isomorphic to $\prod_{n} S_{M_{n}}$. But the only simple non-trivial Polish groups of this form are $S_{\infty}$ and $\mathbb{Z}_{2}$.

Let $(X, d)$ and $(Y, \rho)$ be metric spaces. A function $\pi: X \rightarrow Y$ is called a metric quotient function if it is surjective and for all $y, y^{\prime} \in Y$

$$
\rho\left(y, y^{\prime}\right)=\inf \left\{d\left(x, x^{\prime}\right): x, x^{\prime} \in X, \pi(x)=y \text { and } \pi\left(x^{\prime}\right)=y^{\prime}\right\} .
$$

If $\pi$ is as above, we call $y, y^{\prime} \in Y$ conjugate with respect to $\pi$ if there are $x, x^{\prime} \in X$ such that $\pi(x)=y, \pi\left(x^{\prime}\right)=y^{\prime}$ and $\rho\left(y, y^{\prime}\right)=d\left(x, x^{\prime}\right)$. 
Theorem 4.2. Let $(X, d)$ be a separable ultrametric space. There exists an ultrametric space $(\Omega, \rho)$ and a metric quotient function $\pi: X \rightarrow \Omega$ such that

(i) $\pi$ is invariant under $\operatorname{Iso}(X)$;

(ii) if $\phi$ is a partial isometry of $X$ with finite domain and such that for each $x$ in its domain $\pi(x)=\pi(\phi(x))$, then $\phi$ can be extended to an element of $\operatorname{Iso}(X)$.

Additionally,

(iii) if $y \in \Omega$ is conjugate with respect to $\pi$ to all points in $\Omega$, then each isometry of $\pi^{-1}(y)$ can be extended to an element of $\operatorname{Iso}(X)$.

Theorem 4.2 refines results announced by Feinberg in [3]. For more on this see the first remark below.

By a homogeneity component of a metric space $X$ we mean an orbit under the action of $\operatorname{Iso}(X)$ on $X$. Recall that a metric space is ultrahomogeneous if each partial isometry of the space that has finite domain can be extended to an isometry of the whole space.

We would like to make some remarks on the theorem above.

1. By (i) and (ii) of Theorem 4.2, fibers $\pi^{-1}(y), y \in \Omega$, are precisely homogeneity components of $X$. The idea of the quotient space whose elements are homogeneity components of an ultrametric space $X$ is present in [3]. It is announced in [3, Theorem 3] that, under the assumption that the ultrametric space $(X, d)$ be complete, the space of homogeneity components is itself an ultrametric space with the distance between two homogeneity components $\lambda_{1}, \lambda_{2}$ given by $\inf \left\{d\left(x_{1}, x_{2}\right): x_{1} \in \lambda_{1}, x_{2} \in \lambda_{2}\right\}$. The quotient space is studied further in [3] for compact $X$.

2. By (iii) of Theorem 4.2, fibers $\pi^{-1}(y), y \in \Omega$, are ultrahomogeneous. In particular, if $X$ is assumed to be Polish, fibers are ultrahomogeneous Polish ultrametric spaces and they are completely classified using the methods (if not the results) of [1], [2], and [8, pp.88-89,218-221], as follows. Let $I \subseteq \mathbb{R}_{>0}$ be countable, and let $m: I \rightarrow(\mathbb{N} \backslash\{0\}) \cup\{\infty\}$. Then $I$ and $m$ determine an ultrametric space with the underlying set given by

$$
\begin{aligned}
\{f: I & \rightarrow \mathbb{N}: \\
& \forall i \in I(f(i)<m(i) \text { and }\{j \in I: j>i \text { and } f(j)>0\} \text { is finite })\},
\end{aligned}
$$

and with the ultrametric defined for $f_{1} \neq f_{2}$ by

$$
d\left(f_{1}, f_{2}\right)=\max \left\{i \in I: f_{1}(i) \neq f_{2}(i)\right\} .
$$

All ultrahomogeneous Polish ultrametric spaces are of the above form.

3. Point (ii) of Theorem 4.2 implies that for any $y \in \Omega$, the range of the natural continuous homomorphism

$$
\operatorname{Iso}(X) \ni \phi \rightarrow \phi \uparrow \pi^{-1}(y) \in \operatorname{Iso}\left(\pi^{-1}(y)\right)
$$


is dense in $\operatorname{Iso}\left(\pi^{-1}(y)\right)$ and acts ultrahomogeneously on $\pi^{-1}(y)$. Point (iii), further, gives that this homomorphism is a surjection provided $y$ is conjugate to all points in $\Omega$. For more on this see Example 4.5 below.

We state now a corollary that is somewhat surprising (but that can also be deduced using ideas from [1], [8, pp.88-89]). Note that if a separable ultrametric space $X$ has a dense homogeneity component, then by point 1 above $\Omega$ in Theorem 4.2 is a one point set; thus, there is only one homogeneity component. Therefore, the following corollary follows from point 2 above.

Corollary 4.3. Let $X$ be a separable ultrametric space. If there is a point whose orbit under the action of $\operatorname{Iso}(X)$ is dense, then $X$ is ultrahomogeneous.

Let us call a set $A \subseteq X$ satisfying

$$
d\left(a, a^{\prime}\right)=r \text { for every distinct } a, a^{\prime} \in A
$$

an $r$-isosceles polygon or, shortly, an $r$-polygon. If it is maximal, we will call it a maximal r-polygon.

Lemma 4.4 below is a technical fact that will be important in the proof of Theorem 4.2. After proving the lemma we realized that a related, though weaker, result was proved in [1] using a similar argument. A good source of information on this topic is [8, Sections $6.4,10.6]$.

Lemma 4.4. Suppose that $X$ is an ultrametric space and $\phi: A \rightarrow B$ an isometry between finite subsets of $X$ such that, for every $a \in A, r>0$ and a homogeneity component $Y$ of $X$, we have

$$
\operatorname{dist}(a, Y)<r \Leftrightarrow \operatorname{dist}(\phi(a), Y)<r .
$$

Then for any $x \in X$, there is some $x^{\prime} \in X$ such that $\phi \cup\left\{\left(x, x^{\prime}\right)\right\}$ is an isometry satisfying (12).

Proof. Fix $x \in X$ and let $r=\operatorname{dist}(x, A)$ and let $Y$ be the homogeneity component containing $x$. Let $C$ be an $r$-polygon in $Y$ that is maximal such that for every $c \in C$ there is $a \in A$ with $d(c, a)<r$. For every $c \in C$, fix $c^{\prime} \in Y$ such that

$$
d(c, a)<r \Leftrightarrow d\left(c^{\prime}, \phi(a)\right)<r .
$$

By our assumption, $C^{\prime}=\left\{c^{\prime}: c \in C\right\}$ is maximal such that for every $c^{\prime} \in C^{\prime}$ there is some $b \in B$ with $d\left(c^{\prime}, b\right)<r$ and, as $y$ witnesses that $C$ is not maximal in $Y, C^{\prime}$ is not maximal in $Y$ either. Thus, there exists $x^{\prime} \in Y$ with $\operatorname{dist}\left(x^{\prime}, B\right)=r$.

This $x^{\prime}$ is as required, that is, for each $a \in A, d(x, a)=d\left(x^{\prime}, \phi(a)\right)$. Reason: by the choice of $r$ and $C$, we have two cases

either $d(a, c)<r$ for some $c \in C$; then $d\left(\phi(a), c^{\prime}\right)<r$; and we get $d(x, a)=r=d\left(x^{\prime}, \phi(a)\right)$; 
or $d(a, c)>r$ for all $c \in C$; then $d(\phi(a), c)>r$ for all $c^{\prime} \in C^{\prime}$; and $d(x, a)=d(a, c)=d\left(\phi(a), c^{\prime}\right)=d\left(x^{\prime}, \phi(a)\right)$.

Also, since $x$ and $x^{\prime}$ are in the same component, they satisfy

$$
\operatorname{dist}(x, Y)<r \Leftrightarrow \operatorname{dist}\left(x^{\prime}, Y\right)<r
$$

for every component $Y$ of $X$.

Proof of Theorem 4.2. First we note that every homogeneity component of $X$ is closed. To see this let $Y$ be a homogeneity component, let $a \in Y$, and let $a^{\prime}$ be an element of the closure of $Y$. Set $\phi(a)=a^{\prime}$. This $\phi$ fulfills the assumption of Lemma 4.4. By a standard application of the back-andforth method, using Lemma 4.4, we can extend this $\phi$ to an isometry whose domain and range are countable and dense in $X$. This isometry then easily extends to an element of $\operatorname{Iso}(X)$. Thus, $a^{\prime}$ is in the homogeneity component of $a$, that is, in $Y$.

Using the fact that homogeneity components are closed and the fact that elements of $\operatorname{Iso}(X)$ are isometries, we see that if $Y_{1}, Y_{2}$ are two distinct homogeneity components, then $\operatorname{dist}\left(Y_{1}, Y_{2}\right)>0$. Therefore, we can define a ultrametric on the quotient space $\Omega=X / \sim$, where $\sim$ is the equivalence relation of being in the same homogeneity component, by

$$
\rho\left([x]_{\sim},\left[x^{\prime}\right]_{\sim}\right)=\operatorname{dist}\left([x]_{\sim},\left[x^{\prime}\right]_{\sim}\right) .
$$

It remains to check the ultrametric inequality for $\rho$. This uses the fact that Iso $(X)$ acts transitively on each $\pi^{-1}(y), y \in \Omega$, but is routine and we leave it to the reader.

Note that the mapping $\pi: X \rightarrow \Omega$ defined by $x \mapsto[x]_{\sim}$ is clearly a metric quotient function and is invariant under isometries of $X$, that is, (i) holds.

We show now property (ii), that is, we need to see that every isometry $\phi$ : $A \rightarrow B$ between finite subsets of $X$ that respects homogeneity components of $X$ can be extended to an isometry of $X$. Again this is done by the back-and-forth argument as above using Lemma 4.4 .

To prove property (iii), let us start with the following observation. Suppose that $\phi: Y \rightarrow Y$ is an isometry of a homogeneity component $Y$ of $X$, and that $\phi^{\prime}:(A \cup B) \rightarrow C$ is an isometry satisfying the following conditions:

- the sets $A \subseteq Y, B \subseteq(X \backslash Y)$ are finite, and $\phi^{\prime} \uparrow A=\phi \uparrow A$;

- the mapping $\phi^{\prime}$ respects homogeneity components of $X$;

- we have

$$
\forall b \in B \exists a \in A d(b, a)=\operatorname{dist}(b, Y) .
$$

We claim that, for any $A^{\prime}$ with $A \subseteq A^{\prime} \subseteq Y$, the mapping $\phi^{\prime \prime}=\phi^{\prime} \cup\left(\phi \uparrow A^{\prime}\right)$ is also an isometry. 
Indeed, for a given $b \in B$, fix $a \in A$ with $d(b, a)=\operatorname{dist}(b, Y)$, and let $a^{\prime} \in A^{\prime}$. By our choice of $a$ and the fact that any three points can realize only two distances in an ultrametric space, we have $d\left(\phi^{\prime \prime}(b), \phi^{\prime \prime}\left(a^{\prime}\right)\right)=$ $d\left(b, a^{\prime}\right)$ if $d(b, a) \neq d\left(a, a^{\prime}\right)$. If $d(b, a)=d\left(a, a^{\prime}\right)$, then $d\left(\phi^{\prime \prime}(b), \phi^{\prime \prime}\left(a^{\prime}\right)\right) \leq$ $d\left(\phi^{\prime \prime}(b), \phi^{\prime \prime}(a)\right)$. Since $b$ and $\phi^{\prime \prime}(b)$ are in the same component, we must have

$$
d\left(\phi^{\prime \prime}(b), \phi^{\prime \prime}\left(a^{\prime}\right)\right) \geq \operatorname{dist}\left(\phi^{\prime \prime}(b), Y\right)=d\left(\phi^{\prime \prime}(b), \phi^{\prime \prime}(a)\right),
$$

so

$$
d\left(\phi^{\prime \prime}(b), \phi^{\prime \prime}\left(a^{\prime}\right)\right)=d\left(\phi^{\prime \prime}(b), \phi^{\prime \prime}(a)\right)=d(b, a)=d\left(b, a^{\prime}\right) .
$$

Now, to see that we can extend isometries of $Y$ to isometries of $X$, fix a countable set $\left\{x_{n}: n \in \mathbb{N}\right\} \subseteq(X \backslash Y)$ dense in $X \backslash Y$ and a countable set $\left\{y_{n}: n \in \mathbb{N}\right\} \subseteq Y$ dense in $Y$. For a given $\phi \in \operatorname{Iso}(Y)$, we put $\Phi_{-1}=\emptyset$, $k_{-1}=0$ and proceed as follows.

At step $2 n$, we first extend $\Phi_{2 n-1}$ to an isometry $\Phi_{2 n}^{\prime}$ given by

$$
\Phi_{2 n}^{\prime}=\Phi_{2 n-1} \cup \phi \uparrow\left\{y_{0}, \ldots, y_{k_{2 n}}\right\}
$$

for $k_{2 n}>k_{2 n-1}$ such that there exists $i \leq k_{2 n}$ with $\operatorname{dist}\left(x_{n}, Y\right)=d\left(x_{n}, y_{i}\right)$. Then, applying Lemma 4.4, we find $x^{\prime} \in X$ such that $\Phi_{2 n}=\Phi_{2 n}^{\prime} \cup\left\{\left(x_{n}, x^{\prime}\right)\right\}$ is an isometry. Note that $\Phi_{2 n}$ satisfies (13) for $A=\left\{y_{0}, \ldots, y_{k_{2 n}}\right\}$ and $B=\left\{x_{0}, \ldots, x_{n}\right\}$.

At step $2 n+1$, we extend $\Phi_{2 n}$ to $\Phi_{2 n+1}^{\prime}$ :

$$
\Phi_{2 n+1}^{\prime}=\Phi_{2 n} \cup \phi \uparrow\left\{y_{0}, \ldots, y_{k_{2 n+1}}\right\},
$$

where $k_{2 n+1}>k_{2 n}$ is such that there exists $i \leq k_{2 n+1}$ with $\operatorname{dist}\left(x_{n}, Y\right)=$ $d\left(x_{n}, \phi\left(y_{i}\right)\right)$. By Lemma 4.4 again, we extend $\Phi_{2 n+1}^{\prime}$ to an isometry $\Phi_{2 n+1}=$ $\Phi_{2 n+1}^{\prime} \cup\left(x^{\prime}, x_{n}\right), x^{\prime} \in X$.

In the end, we take $\Phi$ to be the unique extension of $\bigcup_{n} \Phi_{n}$ to an isometry of $X$. Obviously, $\Phi$ extends $\phi$ to an isometry of $X$.

The following example complements point (iii) of Theorem 4.2.

Example 4.5. There exists a Polish ultrametric space with two homogeneity components and an isometry of one of the homogeneity components that does not extend to an isometry of the whole space.

We introduce an ultrametric on the family of all subsets of $\omega \cdot 2=\omega+\omega$ as follows. (Here and below, $\omega$ stands for the first infinite ordinal.) First define for $x, y \subseteq \omega \cdot 2$

$$
\begin{aligned}
& \rho_{1}(x, y)=\min (x \triangle y) \text { if } x \neq y \\
& \rho_{1}(x, y)=\omega \cdot 2 \text { if } x=y .
\end{aligned}
$$

Let

$$
(\omega \cdot 2+1) \ni \alpha \rightarrow \alpha^{*} \in \mathbb{R}
$$


be an order reversing continuous injection with $(\omega \cdot 2)^{*}=0$. Define the ultrametric by letting

$$
d(x, y)=\rho_{1}(x, y)^{*} .
$$

Let $X$ be the set of all subsets $x$ of $\omega \cdot 2$ with $x \cap \omega$ finite. Let $Y$ be the set of all finite subsets of $\omega$. Note that the metric defined above makes $X$ and $Y$ into ultrametric spaces with $X$ separable, complete and with no isolated points and with $Y$ countable and discrete. We treat $X$ and $Y$ as disjoint spaces and define an ultrametric on their union $X \cup Y$ as follows. Let $z_{0}$ be an infinite and coinfinite subset of $\omega$ (say, $z_{0}$ is the set of all even natural numbers). First we extend $\rho_{1}$ from (14). For $x, y \in X$ and $x, y \in Y, \rho_{2}(x, y)$ is defined to be equal to $\rho_{1}(x, y)$; for $x \in X$ and $y \in Y$ put

$$
\rho_{2}(x, y)=\rho_{1}\left(x, y \triangle z_{0}\right) .
$$

Define the ultrametric on $X \cup Y$ to be $\rho_{2}^{*}$.

For $y_{1}, y_{2} \in Y$ the following isometry of $X \cup Y$ carries $y_{1}$ to $y_{2}$

$$
\begin{aligned}
& Y \ni y \rightarrow y \triangle\left(y_{1} \triangle y_{2}\right) \\
& X \ni x \rightarrow x \triangle\left(y_{1} \triangle y_{2}\right) .
\end{aligned}
$$

For $x_{1}, x_{2} \in X$ this is done by the following isometry

$$
\begin{aligned}
& Y \ni y \rightarrow y \triangle\left(\left(x_{1} \triangle x_{2}\right) \cap \omega\right) \\
& X \ni x \rightarrow x \triangle\left(x_{1} \triangle x_{2}\right) .
\end{aligned}
$$

Since points in $Y$ are isolated and those in $X$ are not, each isometry of $X \cup Y$ maps $X$ to $X$ and $Y$ to $Y$. It follows that $X$ and $Y$ are the homogeneity components of $X \cup Y$.

We claim now that there exists an isomerty of $Y$ that cannot be extended to an isometry of $X \cup Y$. Let us identify $Y$ with elements of $2^{\omega}$ that are eventually equal to 0 . Define $\phi: Y \rightarrow Y$ by letting

$$
\begin{aligned}
& \phi(s)(i)=1-s(i) \text { if } \forall j<i s(j)=z_{0}(j) \\
& \phi(s)(i)=s(i) \text { otherwise. }
\end{aligned}
$$

Note that since $z_{0}$ is infinite and coinfinite, $\phi$ maps injectively finite subsets of $\omega$ onto finite subsets of $\omega$, that is, $\phi$ maps $Y$ to $Y$ bijectively. One checks that $\phi$ is, in fact, an isometry.

We claim that $\phi$ cannot be extended to an isometry of $X \cup Y$. Let $\Phi$ be such a hypothetical extension. Fix $n \in \omega$ and consider $x_{n}=z_{0} \cap n$, $y_{n}=\phi\left(x_{n}\right)$. Observe that

$$
\left(x_{n} \triangle z_{0}\right) \cap n=\emptyset, n \subseteq\left(y_{n} \triangle z_{0}\right) .
$$

If we let $\emptyset_{X}$ stand for the point in $X$ that is the empty set, we have

$$
\rho_{2}\left(\Phi\left(\emptyset_{X}\right), y_{n}\right)=\rho_{2}\left(\emptyset_{X}, \Phi^{-1}\left(y_{n}\right)\right)=\rho_{1}\left(\emptyset, x_{n} \triangle z_{0}\right) \geq n-1,
$$


by the definition of $\rho_{1}, \rho_{2}$ and the above observation. Using (15), we now get

$$
\rho_{1}\left(\Phi\left(\emptyset_{X}\right), y_{n} \triangle z_{0}\right)=\rho_{2}\left(\Phi\left(\emptyset_{X}\right), y_{n}\right) \geq n-1 .
$$

This and the fact that $n \subseteq y_{n} \triangle z_{0}$ imply that $n \subseteq \Phi\left(\emptyset_{X}\right)$. Since $n \in \omega$ was arbitrary, we get that $\omega \subseteq \Phi\left(\emptyset_{X}\right)$, which is impossible.

\section{REFERENCES}

[1] F. Delon, Espaces ultramétriques, J. Symbolic Logic 49 (1984), 405-424.

[2] V.Z. Feinberg, Complete homogeneous ultrametric spaces, Dokl. Akad. Nauk SSSR 208 (1973), 1303-1306 (Soviet Math. Dokl. 14 (1973), 288-291).

[3] V.Z. Feinberg, Auto-isometries of compact ultrametric spaces (Russian), Dokl. Akad. Nauk BSSR 22 (1978), 296-299.

[4] S. Gao, A. Kechris, On the classification of Polish metric spaces up to isometry, Mem. Amer. Math. Soc. 161 (2003), no. 766.

[5] M. Katětov, On universal metric spaces, in General Topology and its Relations to Modern Analysis and Algebra, VI (Prague, 1986), pp. 323-330, Heldermann, 1988.

[6] A. Manoussos, P. Strantzalos, On the group of isometries on a locally compact metric space, J. Lie Theory 13 (2003), 7-12.

[7] J. Melleray, Compact metrizable groups are isometry groups of compact metric spaces, Proc. Amer. Math. Soc. 136 (2008), 1451-1455.

[8] B. Poizat, A Course in Model Theory: An Introduction to Contemporary Mathematical Logic, Springer, 2000.

Department of Mathematics, 1409 W. Green Street, University of Illinois, URBANA, IL 61801, USA

Current address: Institute of Mathematics, Polish Academy of Sciences, Śniadeckich 8, 00-956 Warsaw, Poland

E-mail address: mamalicki@gmail.com

Department of Mathematics, 1409 W. Green Street, University of Illinois, URBANA, IL 61801, USA

E-mail address: ssolecki@math.uiuc.edu 